\title{
Review
}

\section{Progress in Research on Infection Factors in Hemodialysis Patients}

\author{
Hanyu Wang \\ The First Affiliated Hospital, Guangzhou University of Chinese Medicine, Guangzhou, China
}

\author{
Keywords \\ hemodialysis; infection; comprehensive \\ prevention
}

\section{Correspondence}

Hanyu Wang,

E-mail:Why6451@126.com

DOI: 10.1515/ii-2017-0127

\begin{abstract}
Patients with uremic diseases and in the stage of chronic renal failure are mainly treated with hemodialysis, which can extend the life and improve the quality of life of patients. Nevertheless, hemodialysis causes numerous adverse reactions, such as infection. Infection after hemodialysis treatment not only increases the suffering of patients but also increases treatment costs and risks. Therefore, hemodialysis complications are a serious global problem and have received extensive attention from clinicians and researchers. This paper provides a comprehensive description of the infection factors of hemodialysis patients. This review aims to provide the basis and guidance for controlling and preventing infection in hemodialysis patients.
\end{abstract}

Infection rates among dialysis patients increase due to repeated blood transfusions and extracorporeal circulation operation coupled with low immunity. In a 16-year follow-up study of 532 hemodialysis patients, Mail-loux et al. ${ }^{[1]}$ found that $36 \%$ of deaths resulted from infection. Furthermore, infection is the second cause of death in dialysis patients with terminal renal failure. The causes for infection during hemodialysis are varied and are mainly classified as the patient's inherent factors and iatrogenic factors. These two classes of factors can exist individually and interact with each other.

\section{Patient's own factors}

\section{Low immunity}

The long-term reduction in food intake and insufficient dialysis may cause gastrointestinal dysfunction in patients with maintenance hemodialysis. At the same time, some nutrients are lost during dialysis, easily causing malnutrition. Over long-term dialysis, the skin and mucous membranes, which act as the immune barrier, become dry and shrink, thus weakening the barrier effect. Thus, the patient becomes susceptible to bacterial infection. Some patients with low cellular immune function are susceptible to lung and intestinal infections. Full dialysis improves the patient' s resistance by decreasing the accumulation of toxins in the body and attenuating the damage caused by toxins on lymphocyte and granulocyte functions.

\section{Protopathy}

Studies have confirmed the high incidence rate of infections in hemodialysis patients with different underlying diseases, such as anemia, chronic glomerulonephritis, electrolyte imbalance, and rheumatoid arthritis, but not in patients suffering from heart disease, high blood pressure, diabetes, diabetic nephropathy, and other diseases ${ }^{[2]}$.

\section{Other factors}

The infection rate of dialysis patients is affected by other factors, such as the patient's age, lifestyle, and psychological status. The incidence rate of infection in the elderly is higher than that in young people. Elderly patients undergoing hemodialysis easily suffer from various infections because of their low immune function. Thus, elderly patients experience a high incidence of opportunistic infection. Elderly dialysis patients are more likely to suffer from malnutrition than patients of other ages. Malnutrition further increases infection rate, and the poor cardiac reserve function of the elderly causes the high incidence of heart failure. Heart failure and pulmonary blood stasis can increase the probability of pulmonary infection. In addition, the patient's 
bad habits, such as smoking and drinking, will negatively affect the treatment effect. Inappropriate diet will also aggravate existing health conditions, induce infections, and even threaten life. Therefore, to decrease infection rates, patients should maintain healthy diets and exercise to improve their immunity.

\section{latrogenic factors}

Iatrogenic infection is a highly common complication and a main cause of death in hemodialysis patients. Studies have confirmed that the incidence rate of nosocomial infection in hemodialysis wards is high as $14.2 \%-49.2 \%$, and that the hemodialysis department has a high incidence of infection ${ }^{[3]}$. Nosocomial infection occurs through several ways: infection during blood transfusion and in the hemodialysis tube; and contamination of the dialysis fluid, water treatment system, and from medical personnel. The following sections describe the first two kinds of infection factors.

\section{Infection in blood transfusion process}

Given the poor awareness and management of hospital safety by hospital staff, patients can become susceptible to some blood-borne viral diseases, such as HIV, hepatitis B and C, cytomegalovirus, Epstein-Barr virus, and hepatitis-associated virus, as well as to Toxoplasma gondii. The likelihood of infection by various blood-borne viral infections among patients on long-term maintenance hemodialysis is significantly higher than that among healthy individuals. Viral infection seriously threatens the life and health of patients and of the hemodialysis ward staff. Moreover, studies have shown that blood transfusion and hemodialysis duration are independent risk factors for infections in hemodialysis patients. Thus, the need for blood transfusion must be decreased by improving anemia symptoms through treatment with erythropoietin and other drugs. In addition, during blood transfusion, the clinicians must follow strict disinfection measures and exert strict control over blood transfusion and blood products. Patients with viral infections should be isolated as soon as possible. These measures can prevent the infection of hemodialysis patients with blood-borne viral diseases.

\section{Hemodialyzer and piping infection}

The dialyzer is the core of hemodialysis. Given that maintenance hemodialysis is based on the establishment of vascular access, potential infection risks from dialysis equipment should be monitored.

A deep intravenous integrated catheter is widely used in clinical practice given its quick installment with limited damage and good dialysis effect. However, the likelihood of infection also increases with the installment of a deep intravenous catheter. Once a catheter-related infection occurs, the sensitive antibiotic therapies of the whole body or with heparin are adopted immediately but with poor effect. In addition, the injudicious application of antibiotics can cause secondary infection with drugresistant strains or cause severe allergic reactions, which hide the symptoms and signs of infection. Catheter-related infections are often the leading cause of catheter removal or even death. Infection rates with deep intravenous catheters are affected by catheter material, catheter placement, indwelling catheter time, and operation techniques. Studies have confirmed that infection rate with a single lumen catheter is $8.3 \%$ and that with double lumen catheters is as high as $37.3 \%$. There are three catheterization methods for hemodialysis patients - in internal jugular veins, subclavian veins, and femoral veins [4]. These three kinds of catheterization methods have their own advantages and disadvantages, and are selected in accordance with the different circumstances of the patient. Patients with tracheotomy should not receive internal jugular vein catheterization, which is susceptible to sputum contamination; femoral vein catheterization is susceptible to contamination with stool and urine and is difficult to observe. For general patients, placement of the catheter in the subclavian vein should be first considered, and then in the internal jugular vein, and finally in the femoral vein. The duration of catheterization is positively correlated with infection rate. However, if the sterile operation and management of catheterization are ensured, the infection rate of long-term catheterization can be decreased.

\section{Conclusion}

Infection among hemodialysis patients is a very serious and urgent problem that requires resolution. Preventing infection to improve the survival and quality of life of hemodialysis patients requires the joint efforts of the patients and hospital staff. 


\section{Declarations}

\section{Acknowledgements}

No.

\section{Competing interests}

The author declares that he has no competing interest.

\section{Authors' contributions}

HY Wang made the literature analysis and wrote, discussed and revised the manuscript of this review.

\section{References}

1 Mailloux LU, Bellucci AG, Wilkes BM, et al. Mortality in dialysis patients: Analysis of the causes of death. Am J Kidney Dis,1991 (18):326-327.

2 Wei Q. Study on Hemodialysis Patients with Concurrent Infection. Chinese Journal of Nosocomiology, 2004,14 (5): 599 - 600.

3 Wan H. Analysis on Factors of Central Venous Catheter Infection and Status Quo of Nursing Research. Chinese Nursing Research, 2005,19 (9): $1791-1792$.

4 Ma Y. Observation on the Nursing Effect of the Prevention of Catheterrelated Infection in Hemodialysis Patients. Journal of Nursing (China), 2007,14 (6): 53-54. 